LA GRANA:

REVISTA DE

CIENCIAS DE LA VIDA

pISSN:1390-3799; eISSN:1390-8596

http:/ / doi.org/10.17163/lgr.n29.2019.10
Artículo científico / Scientific paper

PRODUCTOS NATURALES

\title{
EVALUACIÓN MICROBIOLÓGICA Y COMPOSICIÓN QUÍMICA DE EXTRACTOS ORGÁNICOS DE Euphorbia aff. viridis (Klotzsch \& Garcke) Boiss SOBRE Staphylococcus Aureus, Klebsiella Pneumoniae Y Escherichia Coli
}

\author{
MICROBIOLOGICAL EVALUATION AND CHEMICAL COMPOSITION OF ORGANIC \\ EXTRACTS FROM Euphorbia aff. viridis (Klotzsch \& Garcke) Boiss ON \\ Staphylococcus Aureus, Klebsiella Pneumoniae AND Escherichia Coli
}

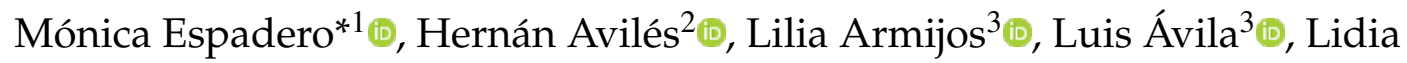 \\ Idrovo $^{2}{ }^{\oplus}$, María Idrovo $^{2}$ y Christian Oyola ${ }^{2}$
}

${ }^{1}$ Laboratorio de Ciencias de la Vida, Universidad Politécnica Salesiana, Cuenca, Ecuador.

${ }^{2}$ Carrera de Biotecnología, Universidad Politécncia Salesiana, Cuenca, Ecuador.

${ }^{3}$ Departamento de Investigación, Jatun Yachay Wasi, Colta, Ecuador.

*Autor para correspondencia: mespadero@ups.edu.ec

Manuscrito recibido el 13 de diciembre de 2018. Aceptado, tras revisión, el 18 de febrero de 2019. Publicado el 1 de marzo de 2019.

\begin{abstract}
Resumen
Euphorbia aff. viridis (Klotzsch Garcke) Boiss. es una especie vegetal perteneciente a la familia Euphorbiaceae, forma parte de las más diversas del continente americano, y es empleada en múltiples tratamientos en medicina ancestral. En el presente estudio se analizaron los compuestos orgánicos extraídos de las hojas de la planta. Se estableció la descripción y clasificación taxonómica de la especie vegetal. Para el estudio fitoquímico se analizaron los fenoles, taninos, saponinas, cumarinas, lactonas y flavonoides, observándose resultados positivos para cada compuesto químico. La caracterización química mediante cromatografía de gases acoplada a espectrometría de Masas GC-MS, mostró 36, 66\% de lanosterol, $12,25 \% \beta$-sitosterol, $5,11 \% 3-\beta$-colest $-4-e n-3-o l, 4,73 \%$ ácido hexadecanoico como elementos mayoritarios. El ensayo microbiológico de extractos etanólicos mostró un porcentaje de inhibición del $44 \%$ sobre Klebsiella pneumoniae (ATCC70693) y $43 \%$ en Escherichia coli (ATCC10536) con una CMI de 30 $\mathrm{l} / \mathrm{ml}$ para cada cepa, y se observó un resultado negativo de inhibición para Staphylococcus aureus (ATCC24213). Los extractos orgánicos de Euphorbia aff. viridis (Klotzsch Garcke) Boiss. presentaron actividad antimicrobiana pudiéndose observar su potencial uso como agentes antimicrobianos.
\end{abstract}

Palabras clave: Antimicrobiano, extracto orgánico, GC-MS, Euphorbia aff. viridis (Klotzsch Garcke) Boiss. 


\begin{abstract}
Euphorbia aff. viridis (Klotzsch Garcke) Boiss. a plant species belonging to the Euphorbiaceae family, is one of the most diverse of the American continent, and is used in multiple treatments in ancestral medicine. In the present study, organic compounds extracted from the leaves of the plant were analyzed. The description and taxonomic classification of the plant was established. For the phytochemical study, phenols, tannins, saponins, coumarins, lactones and flavonoids were analyzed, and positive results were observed for each chemical compound with the exception of alkaloid compounds. The chemical characterization by gas chromatography coupled to mass spectrometry GC-MS, showed $36,66 \%$ lanosterol, $12,25 \% \beta$-sitosterol, $5,11 \% 3-\beta$-cholest $-4-e n-3-o l, 4,73 \%$ hexadecanoic acid as major elements. The microbiological assay of ethanolic extracts showed a $44 \%$ inhibition percentage on Klebsiella pneumoniae (ATCC70693) and $43 \%$ on Escherichia coli (ATCC10536) with an MIC of 30 $\mu \mathrm{l} / \mathrm{ml}$ for each strain, and a negative inhibition result was observed for Staphylococcus aureus (ATCC24213). The organic extracts of Euphorbia aff. viridis (Klotzsch Garcke) Boiss. presented antimicrobial activity being able to observe their potential use as antimicrobial agents.
\end{abstract}

Keywords: Antimicrobial, organic extract, GC-MS, Euphorbia aff. viridis (Klotzsch Garcke) Boiss.

Forma sugerida de citar: $\quad$ Espadero, M., Avilés, H., Armijos, L., Ávila, L., Idrovo, L., Idrovo, M. y Oyola, C. (2019). Evaluación microbiológica y composición química de extractos orgánicos de Euphorbia aff. viridis (Klotzsch \& Garcke) Boiss sobre Staphylococcus Aureus, Klebsiella Pneumoniae y Escherichia Coli. La Granja: Revista de Ciencias de la Vida. Vol. 29(1):119-129. http: //doi.org/10.17163/lgr.n29.2019.10.

\footnotetext{
IDs Orcid:

Mónica Espadero: https:/ /orcid.org/0000-0002-2608-9387

Hernán Avilés: https://orcid.org/0000-0002-0671-4928

Lilia Armijos: https:/ / orcid.org/0000-0003-0802-3701

Luis Ávila: https:/ / orcid.org/0000-0001-6576-2696

Lidia Idrovo: https:/ / orcid.org/0000-0001-9875-9477

María Idrovo: https:/ / orcid.org/0000-0001-9711-0916

Christian Oyola: https:/ / orcid.org/0000-0003-2289-5688
} 


\section{Introducción}

En la población ecuatoriana, el uso de plantas con fines terapéuticos está estrechamente ligado a tradiciones culturales. Actualmente, la etnobotánica ha despertado una conciencia de cambio en las generaciones científicas y académicas, y estudios realizados por Coy, Gómez y Castiblanco, (2016), describen la importancia medicinal, la taxonomía y los usos etnobotánicos de especies del género Euphorbiaceae. La familia Euphorbiaceae comprende alrededor de 8100 especies; es común en países tropicales, y forma parte de las familias más diversas entre las Magnolophytas, después de las Orchidaceas, Asteráceas, Fabáceas, Poáceas y Rubiáceas (Ogbulie y col., 2007; Zegarra, 2015). Esta familia es una de las Angiospermas con más diversidad en cuanto a su hábitat y morfología, varían en tamaño desde los árboles como el Havea con gran altura hasta plantas más pequeñas como los cactus (Al-Mughrabi, 2003; Coy, Gómez y Castiblanco, 2016). Según estudios etnobotánicos realizados por Mwine y Van Damme, (2011) y Oyerma y col., (2010) la familia Euphorbiaceae es empleada con fines medicinales, como la especie Hura crepitans L., que se caracteriza por poseer características astringentes.

En la medicina ancestral, el uso de especies de la familia Euphorbiaceae es variado, pudiendo utilizarse en dermatología, como fungicida y antimicrobiano, para tratamiento de enfermedades gastrointestinales, y enfermedades bacterianas como la gonorrea (Azuaje y col., 2017). Estudios realizados por Sabandar y col., (2013) sobre el género Jatropha han descrito su uso en enfermedades de la piel, como infecciones e inflamaciones.

Euphorbia aff. viridis (Klotzsch \& Garcke) Boiss. es una planta fanerógama, y se multiplica de forma asexual mediante estacas (Giraldo, Ríos y Polanco, 2015). Estudios realizados por Flores Martínez y col., (2012) indican que su siembra se realiza cuando la Luna inicia sus fases (Luna creciente o Luna llena), debido a una mayor incidencia de los campos magnéticos sobre el sistema radicular de la planta, y en función a cada fase lunar, la influencia sobre los tallos y su follaje será mayor hasta el desarrollo final de su inflorescencia. Una vez concluida esta etapa, se realiza la cosecha en luna llena, ya que las partes que conforman la planta se encontraran con un mayor porcentaje de cada uno de sus compuestos químicos constituyentes, pudiéndose emplear potencialmente en medicina tradicional (Torres, 2012).
Las investigaciones y aportes realizados en Ecuador sobre Euphorbia aff. viridis (Klotzsch E Garcke) Boiss. con respecto a sus propiedades medicinales son escasos. Estudios realizados por Bittner y col., (2001) mostraron que Euphorbia peplus, Euphorbia lactiflua, Euphorbia portulacoides y Euphorbia serpens poseen actividad antimicrobiana, siendo un indicativo para que Euphorbia aff. viridis (Klotzsch $\mathcal{E}$ Garcke) Boiss. tenga compuestos químicos con propiedades similares referente a las especies descritas.

En este estudio se propuso relacionar las propiedades terapéuticas propias del extracto con la actividad biológica contra agentes microbianos (Zampini, Cudmani e Isla, 2007), es por ello que se utilizaron diferentes técnicas para el análisis fitoquímico y la actividad antimicrobiana (López Ramosa y col., 2016), sobre Staphylococcus aureus (ATCC24213), Klebsiella pneumoniae(ATCC70693) y Escherichia coli(ATCC10536). En el presente estudio se analizaron los compuestos presentes en Euphorbia aff. viridis (Klotzsch $\mathcal{E}$ Garcke) Boiss. siguiendo los estudios de Suzuki y col., (2006), dando las pautas necesarias para el aprovechamiento de esta especie vegetal que crece en Ecuador, para su posterior uso en medicina ancestral.

\section{Materiales y métodos}

La identificación taxonómica de la especie se lo realizó el 9 de agosto del 2017 por medio de un curador del Herbario de la Universidad del Azuay, en Cuenca, Ecuador.

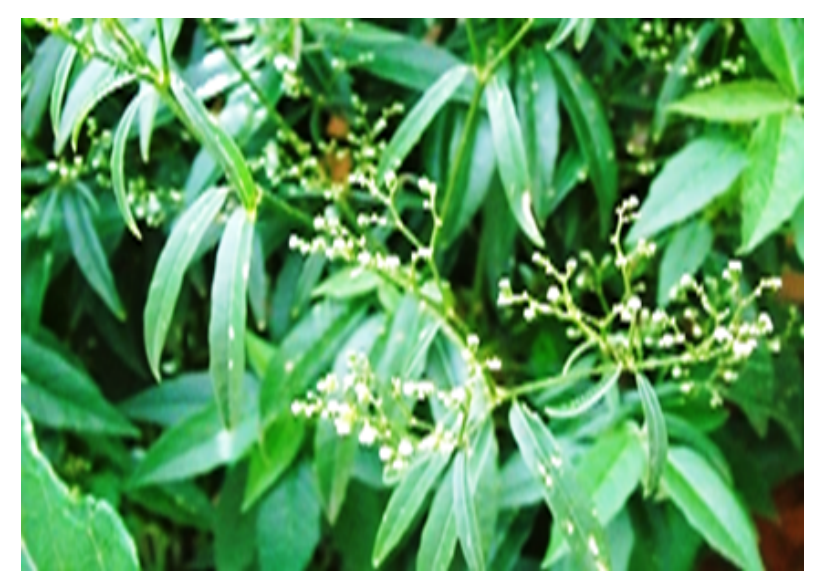

Figura 1. Planta Euphorbia aff. viridis (Klotzsch \& Garcke) Boiss. con Flor 


\subsection{Recolección de material veguetal}

Se recolectaron las hojas de la planta Euphorbia aff. viridis (Klotzsch Garcke) Boiss. en el Cantón Cuenca, Provincia del Azuay, Ecuador (Long: -79.0093518 Lat: -289014096), mediante el uso de tijeras de podar Stanley Steel, posteriormente fueron recolectadas en recipientes de Isopropileno y trasportadas a los Laboratorios de Ciencias de la Vida de la Universidad Politécnica Salesiana.

\subsection{Obtención de extractos}

Las hojas frescas de Euphorbia aff. viridis (Klotzsch $\mathcal{E}$ Garcke) Boiss. fueron secadas a $60^{\circ} \mathrm{C}$ por un periodo de ocho (8) días, y mediante presión mecánica se pudieron obtener partículas finas. La cantidad de materia vegetal que se utilizó fue de $50 g$ para extracto seco como para extracto fresco. Posteriormente, se obtuvo $10 \mathrm{ml}$ de extracto mediante maceración con etanol absoluto, eliminando el solvente utilizando un evaporador rotativo TECNAL TR-211 (Hernández Ocura y col., 2014; González Villa, 2004; Muhammad Abubakar, 2009).

\subsection{Análisis fitoquímico}

Se realizó la determinación y presencia de fenoles y taninos, saponinas, cumarinas y lactonas, flavonoides, quinonas y alcaloides en el extracto obtenido según López Ramos y col., (2017).

\subsection{Cromatografía de gases-espectrometría de masas GC-MS}

Se utilizó un cromatógrafo de gases Agilent modelo GC-6890 acoplado a un espectrómetro de masas cuadrupolar HP 5973N. La inyección de la muestra se realizó por el modo "split" con una relación 1:10 (Parrales, Reyes y Pine Tobar, 2012), siendo la temperatura del inyector $280^{\circ} \mathrm{C}$. Se trabajó con una temperatura inicial de $80^{\circ} \mathrm{C}$, incrementándose hasta $310^{\circ} \mathrm{C}$, seguido de un proceso isotérmico de $20 \mathrm{mn}$ (García Luján y col., 2010). El volumen final de inyección fue de $1 \mu l$. Los componentes de la muestra se fraccionaron en la columna HP-5MS. El tiempo total de elución fue de 100 minutos, operado por ionización electrónica a $70 \mathrm{eV}$ con un rango de masas de 35-700uma. Como gas portador se utilizó helio a un flujo de $0,8 \mathrm{ml} / \mathrm{min}$.

\subsection{Estudio microbiológico}

Se utilizó la técnica de la difusión en agar empleando discos, basados en los lineamientos y protocolos establecidos por Clinical Laboratory Standars Institute (CLSI) y la Organización Mundial de la Salud (OMS). Existe un fundamento en la relación entre la concentración del antibiótico a utilizar y la formación de halos de inhibición que éste produce sobre la superficie de la placa que contiene el medio de cultivo (Ramírez, Castillo y Vargas, 2013). Se utilizó extracto de hoja seca y extracto de hoja fresca en concentraciones de extracto puro (100\%), 80/20 y 50/50 etanol-agua.

\subsection{Evaluación de actividad antimicrobia- na}

Se pesó $0,03 g$ del extracto crudo y se solubilizó con $1 \mathrm{ml}$ de Dimetilsulfóxido (DMSO) de grado analítico para lograr una concentración de $30 \mathrm{mg} / \mathrm{ml}$. Para la activación de los microorganismos: Staphylococcus aureus (ATCC24213), Klebsiella pneumoniae (ATCC70693) y Escherichia coli (ATCC10536), se aislaron las bacterias en agar nutritivo de marca Acumedia, preparado según especificaciones de la casa matriz y esterilizada en autoclave a $121^{\circ} \mathrm{C}$ y 1 atmosfera durante $15 \mathrm{~min}$.

El procedimiento se realizó mediante la técnica de agotamiento de la muestra por estría, en cajas petri, incubadas durante $18-24$ horas a $37^{\circ} \mathrm{C}$. Concluido el tiempo de incubación, con ayuda de un asa bacteriológica estéril, se tomaron de 3 a 5 colonias morfológicamente similares y se suspendieron en una solución salina $\mathrm{NaCl}$ al $0,9 \%$ hasta alcanzar la turbidez comparable con el patrón 0,5 de McFarland, de manera cualitativa.

Para el ensayo de actividad antibacteriana se empleó agar Muller-Hilton de marca Difco ${ }^{T M}$, este fue colocado en cajas petri, las cuales fueron impregnadas con $100 \mu \mathrm{l} /$ caja con la suspensión ajustada de cada bacteria indicadora, también se emplearon discos estériles de $6 \mathrm{~mm}$ de diámetro, humedecidos con $10 \mu l$ de extracto diluido; como control positivo se usó cloranfenicol en concentración de $100 \mu \mathrm{l} / \mathrm{ml}$ y como control negativo se empleó DMSO. Seguidamente las cajas petri se incubaron a $37^{\circ} \mathrm{C}$ por 24 horas; cada uno de los ensayos se realizó por triplicado (Hammer, Carson y Riley, 1999).

El porcentaje de inhibición se calculó mediante la fórmula (1), teniendo como referencia la medición 
del diámetro de la zona de inhibición del control positivo y la medición del halo de los extractos testeados (Corzo Barragán, 2012). Donde $\oslash$ halo extracto, es el diámetro del halo formado por el disco que contiene el extracto; $\oslash$ halo blanco, es el diámetro del halo formado por el disco del control negativo y $\oslash$ halo control positivo, es el diámetro del halo formado por el disco con el control positivo.

$$
\% \text { Inhibición }=\frac{(\oslash \text { halo extracto }-\oslash \text { halo blanco })}{(\oslash \text { halo control positivo }-\oslash \text { halo blanco })} \times 100
$$

\subsection{Determinación de la concentración mí- nima inhibitoria (CMI)}

La concentración mínima inhibitoria (CMI), se aplicó para determinar la menor concentración de cada uno de los extractos que inhibe el crecimiento de las bacterias. El punto final (CMI) se considera la menor concentración de compuesto frente a la cual el microorganismo ensayado no presenta desarrollo visible (Cruz, Rodríguez y Rodríguez, 2010). La CMI, fue evaluada solo para extractos que evidenciaron inhibición frente a las bacterias de referencia, y se realizó por el método de dilución en agar TSB (marca Acumedia), en donde se incorporó el extracto a evaluar en el medio con agar. La técnica fue realizada en micro placas con fondo plano de 24 pocillos, en las cuales se dispusieron diferentes volúmenes de solución de los extractos en cada pocillo y se realizaron micro diluciones con el medio de cultivo.

Las concentraciones probadas fueron $30 \mu \mathrm{l} / \mathrm{ml}$, $15 \mu \mathrm{l} / \mathrm{ml}, \quad 7,5 \mu \mathrm{l} / \mathrm{ml}, \quad 3,75 \mu \mathrm{l} / \mathrm{ml}, \quad 1,875 \mu \mathrm{l} / \mathrm{ml} \quad$ у
$0,9375 \mu \mathrm{l} / \mathrm{ml}$ para K. pneumoniae, y desde $60 \mu \mathrm{l} / \mathrm{ml}$ para E. coli. En cada pozo se sembraron $2 \mu l$ de Klebsiella pneumoniae (ATCC70693) y Escherichia coli (ATCC10536) a una concentración de 1,5 × 106 $\mathrm{UFC} / \mathrm{ml}$, comparables al tubo 0,5 en la escala de turbidez de McFarland. Después de 24 horas de incubación a $37^{\circ} \mathrm{C}$, las microplacas se examinaron visualmente.

\section{Resultados}

La cantidad obtenida de extracto seco fue de $14,82 \mathrm{ml}$ y de extracto fresco $20,44 \mathrm{ml}$. En la tabla 1 se muestra el tamizaje fitoquímico correspondiente al extracto obtenido a partir de Euphorbia aff. viridis (Klotzsch Garcke) Boiss. y solución de etanol al $100 \%$. En la Tabla 2 se recogen los resultados de la presencia de Alcaloides en el extracto analizado. El análisis de cromatografía de gases acoplado a espectrometría de masas GC-MS, pudo evidenciar los resultados expuestos en la Tabla 3.

Tabla 1. Resultados de tamizaje fitoquímico.

\begin{tabular}{cc}
\hline Componentes & Resultados \\
\hline Ensayo de Fenoles y Taninos & Positivo \\
Ensayo de Cumarinas y Lactonas & Positivo \\
Ensayo de Quinonas & Positivo \\
Ensayo de Saponinas & Positivo \\
Ensayo de Catequinas & Positivo \\
\hline
\end{tabular}

Tabla 2. Resultados de Ensayo de Alcaloides

\begin{tabular}{cc}
\hline Ensayo & Resultados \\
\hline Ensayo de Dragendorff & Negativo \\
Ensayo de Wagner & Negativo \\
Ensayo de Mayer & Negativo \\
\hline
\end{tabular}


PRODUCTOS NATURALES Espadero, M., Avilés, H., Armijos, L., Ávila, L., Idrovo, L., Idrovo, M. y Oyola, C.

Tabla 3. Resultados de Ensayo de Cromatografía de masas.

\begin{tabular}{llll}
\hline No. & Tr & AbundanciaRelativa \% & Compuesto \\
\hline 1 & 5722 & 0,92 & Ácido benzoico \\
2 & 6623 & 0,52 & Glicerol \\
3 & 21499 & 1,46 & Éster etílico del ácido hexadecanoico \\
4 & 22547 & 4,73 & Ácido hexadecanoico \\
5 & 24905 & 10,40 & $3,7,11,15$-tetrametill-2-hexadecenol \\
6 & 25417 & 0,81 & Ácido- 9,12 - octadecenoico \\
7 & 25529 & 2,26 & Ácido - $\alpha$-linoleico \\
8 & 26006 & 1,20 & Ácido octadecanoico \\
9 & 38944 & 5,49 & Desconocido \\
10 & 40298 & 36,66 & Lanosterol \\
11 & 40852 & 13,72 & Lanosterol (isómero) \\
12 & 41213 & 12,15 & $\beta$-sitosterol \\
13 & 41608 & 2,91 & $\beta$-amirina \\
14 & 41872 & 1,66 & Cicloartenol \\
15 & 42248 & 5,11 & 3 - $\beta$-colest-4-en-3-ol \\
\hline
\end{tabular}

${ }^{*} \operatorname{Tr}$ : Tiempo de retención en columna.

El análisis antibacteriano frente a los microorganismos aislados: Staphylococcus aureus (ATCC24213), Klebsiella pneumoniae (ATCC70693) y Escherichia coli (ATCC10536), se muestra en la tabla 4 . El estudio fue realizado en base al extracto obtenido de hojas frescas y secas en solución etanólica, dando como resultado que las hojas secas presentan mayor actividad antimicrobiana frente al extracto de hojas frescas. Los extractos estudiados presentaron inhibición frente a Klebsiella pneumoniae (ATCC70693) y Escherichia coli (ATCC10536) siendo negativo para Staphylococcus aureus (ATCC24213), teniendo mejores resultados en los extractos de hojas secas.

Tabla 4. Resultados ante la prueba de actividad antimicrobiana del extracto etanólico.

\begin{tabular}{lllll}
\hline & & $100.00 \%$ & $80 / 20$ & $50 / 50$ \\
\hline Staphylococcus aureus & SECO & $(-)$ & $(-)$ & $(-)$ \\
(ATCC 24213) & FRESCO & $(-)$ & $(-)$ & $(-)$ \\
\hline Klebsiella pneumoniae & SECO & $(+)$ & $(+)$ & $(-)$ \\
(ATCC 70693) & FRESCO & $(+)$ & $(-)$ & $(-)$ \\
\hline \multirow{2}{*}{ Escherichia coli } & SECO & $(+)$ & $(+)$ & $(-)$ \\
& FRESCO & $(+)$ & $(-)$ & $(-)$ \\
\hline
\end{tabular}

(+) Resultado positivo, (-) Resultado negativo.

En las figuras 3 y 4 , se expone de manera porcentual la inhibición del extracto frente a los microorganismos empleados, calculado de acuerdo con el halo de inhibición y teniendo como referencia el control positivo. Siguiendo el protocolo propuesto por Corzo Barragán, (2012), se determinó la CMI a las 24 horas correspondientes al tiempo de observación, teniendo en consideración que la inhibición se realiza en una concentración de $30 \mu \mathrm{l} / \mathrm{ml}$ en ambos estudios. En las Figuras 4 y 5, se presentan los resultados obtenidos de CMI frente a K. pneumoniae y E. coli respectivamente. 


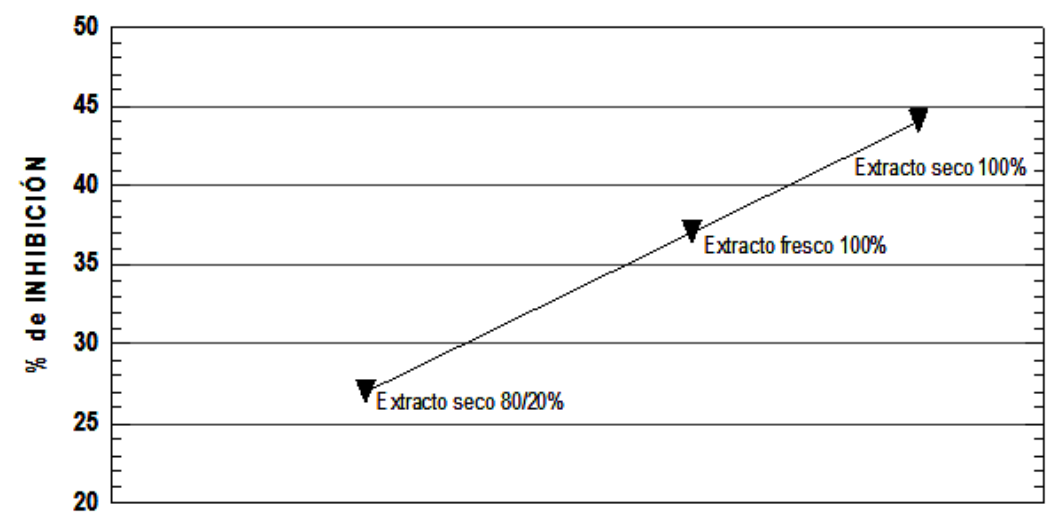

Figura 2. Porcentaje de inhibición de los extractos etanólicos frente a Klebsiella pneumoniae.

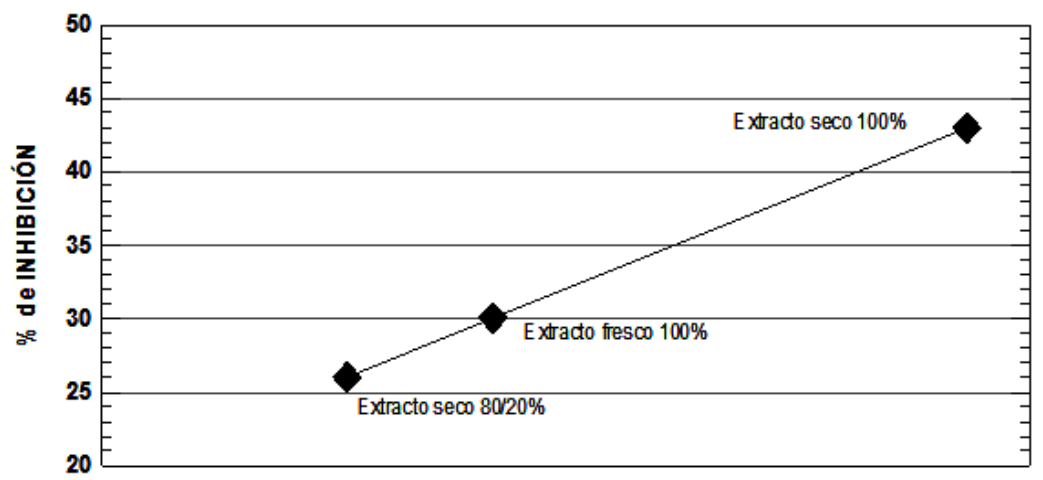

Figura 3. Porcentaje de inhibición de los extractos etanólicos frente a la Escherichia coli.

\section{Discusión}

El extracto alcohólico de hojas secas y frescas de Euphorbia aff. viridis (Klotzsch $\mathcal{E}$ Garcke) Boiss. confirmó la presencia de fenoles, taninos, cumarinas y lactonas, quinonas, saponinas y catequinas, dando control positivo al análisis fitoquímico correspondiente y mostrando similitud con los estudios realizados por Gutiérrez Gaitén y col., (2011), donde lactonas, fenoles, cumarinas y taninos fueron aisladas a partir de extractos hexánicos y de extracto de acetato de etilo. Por otro lado, en el "Estudio fitoquímico de las hojas de la especie vegetal Croton schiedeanus (Euphorbiaceae)" realizado por Chiappe, (2015), se pudo evidenciar la presencia de metabolitos se- cundarios de tipo carotenoide, fenólico, flavonoide y alcaloide, lo que está de acuerdo con la quimio taxonomía de la familia y el género.

No se detectó la presencia de alcaloides en este estudio con relación a varias especies estudiadas del género, cuyo resultado ha sido positivo, como en Bittner y col., (2001), quienes expresan que se aislaron alcaloides del tipo morfina y aporfina. Gomes y col., (2017), realizaron un estudio sobre "Características fisicoquímicas y efecto citotóxico del extracto metanólico de Croton heliotropiifolius Kunth (Euphorbiaceae)", donde el análisis físico-químico realizado mediante TLC, HPLC, FT-IR y UV-Vis, reveló la presencia de flavonoides y la ausencia de alcaloides, cumarinas, saponinas y taninos condensados. 


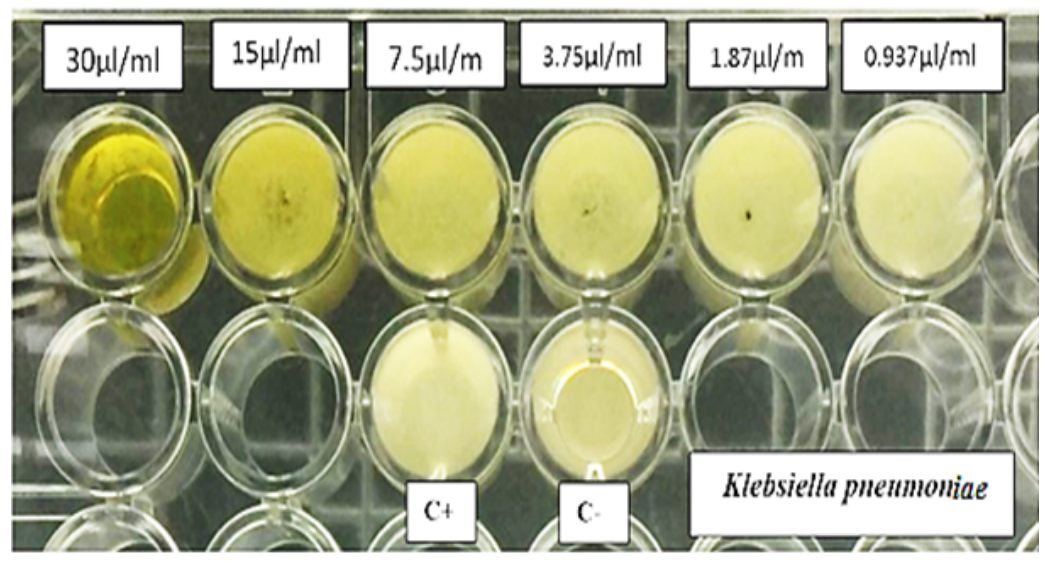

Figura 4. Resultado de la concentración mínima inhibitoria frente a Klebsiella pneumoniae.

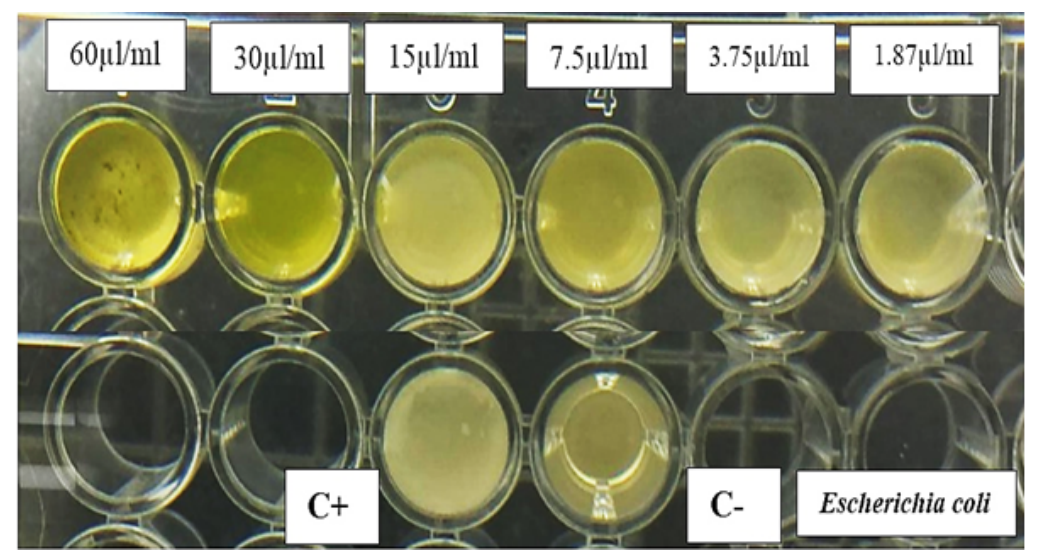

Figura 5. Resultado de la concentración mínima inhibitoria frente a Escherichia coli.

GC-MS es una método muy robusto para en análisis de las moléculas químicas presentes en una especie vegetal, pudiendo establecer sus compuestos mayoritarios así como posibles marcadores químicos para la especie de interés (Valdéz, Delgado y Ramírez, 2018). Con respecto al análisis de GC-MS de Euphorbia aff. viridis (Klotzsch $\mathcal{E}$ Garcke) Boiss., se pudo identificar la presencia de ácido benzoico $0,92 \%$, glicerol $0,52 \%$, éter etílico del ácido hexadecanoico $1,46 \%$, ácido hexadecanoico $4,73 \%, 3,7 ; 11,15-$ tetrametill-2-hexadecenol $10,40 \%$, ácido- 9,12- octadecenoico $0,81 \%$, ácido $-\alpha$-linoleico $2,26 \%$, ácido octadecanoico $1,20 \%$, lanosterol $36,66 \%$, lanosterol (isómero) $13,72 \%, \beta$-sitosterol $12,15 \%, \beta$ amirina $2,91 \%$, cicloartenol $1,66 \%, 3-\beta$-colest- 4 -en3 -ol 5, $11 \%$, siendo el Lanosterol con $36,66 \%$ el compuesto mayoritario.
Rodríguez Pava, Zarate Sanabria y Sánchez Leal, (2017), en "Actividad antimicrobiana de cuatro variedades de plantas contra la importancia clínica de patógenos en Colombia", concluyen que los flavonoides y quinonas poseen propiedades antibacterianas importantes, que al contacto con Streptococcus pneumoniae y Staphylococcus aureus han mostrado actividad sobre dichas cepas, siendo nula para Staphylococcus aureus (ATCC24213) en este estudio. En estudios realizados por Alvarez y Toso, (2018), sobre la especie Euphorbia peplus mostraron resultados positivos para actividad antimicrobiana contra S. aureus, mientras que Euphorbia serpens es efectivo contra E. coli y S. aureus; guardando similitud con los resultados obtenidos en este estudio donde se observó bioactividad del extracto al $100 \%$ sobre Escherichia coli. Corzo Barragán, (2012) y Ogbulie y 
col., (2007), muestran que la familia de las Euphorbiaceas posee actividad antimicrobiana, pudiéndose observar dicha actividad sobre Klebsiella pneumoniae y Escherichia coli en el presente estudio.

\section{Conclusión}

El lanosterol es un compuesto triterpénico tetra cíclico que integra mayoritariamente los extractos orgánicos de Euphorbia aff. viridis (Klotzsch \& Garcke) Boiss. que se desarrolla en la región Andina del Ecuador, y que podrían ser utilizados como marcadores químicos de la especie estudiada. Los extractos orgánicos son compuestos generalmente seguros para su uso en farmacología y cosmetología, donde su actividad antimicrobiana frente a E. coli y K. pneumoniae, demuestran su potencial uso como agente antimicrobiano y para su empleo en la medicina ancestral.

\section{Agradecimientos}

Se agradece a Danilo Minga Ochoa, taxónomo curador del Herbario de la Universidad del Azuay, quien brindó su apoyo para la identificación de la planta. Al departamento de Biotecnología de la Universidad Politécnica Salesiana, Sede Cuenca; Laboratorios de Ciencias de la Vida por el apoyo brindado en el desarrollo de este proyecto y al Ms.C. Adrián Valdez, miembro de grupo de investigación INVIAM por la lectura crítica del artículo.

\section{Referencias}

Al-Mughrabi, Khalil I. (2003). "Antimicrobial activity of extracts from leaves, stems and flowers of Euphorbia macroclada against plant pathogenic fungi». En: Phytopathologia mediterranea 42.3, 245-250. Online: https://bit.ly/2H0a4X7.

Alvarez, Hebe L. y Ricardo E. Toso (2018). «Actividad antimicrobiana, larvicida, antiviral y citotóxica de especies medicinales nativas del género Euphorbia, E. collina Phil. y E. serpens HBK caracterización de los metabolitos bioactivos». En: Ciencia Veterinaria 17.2, 53-54. Online: https: / / bit.ly/2U1NHEb.
Azuaje, Mariangel y col. (2017). "Actividad antifúngica in vitro de extractos de Hura crepitans L.(Euphorbiaceae) frente a Candida albicans Mariangel Azuaje, Silvana Villarreal, Luis Rojas Fermín, Clara Díaz, María Rodríguez». En: Avances en Biomedicina 6.3, 197-202. Online: https:/ / bit.ly/2GUrSCJ.

Bittner, M. y col. (2001). "Estudio quimico de especies de la familia euphorbiaceae en Chile». En: Boletín de la Sociedad Chilena de Química 46.4, 419-431. Online: https:/ / bit.ly/2BLTAy2.

Chiappe, Andrea C. (2015). «Estudio fitoquímico de las hojas de la especie vegetal Croton schiedeanus (Euphorbiaceae)». Tesis doct. Universidad Nacional de Colombia, Online: https: / / bit.ly / 2GY0Omk.

Corzo Barragán, Diana C. (2012). «Evaluación de la actividad antimicrobiana del extracto etanólico». En: Revista mexicana de ciencias farmacéuticas 43.3, 81-86. Online: https:/ / bit.ly/2QQi9Pj.

Coy, Andrés, Diana Gómez y Fabio Andrés Castiblanco (2016). «Importancia medicinal del género Croton (Euphorbiaceae)». En: Revista Cubana de Plantas Medicinales 21.2, 234-247. Online: https:/ / bit.ly/2DVsZ1O.

Cruz, Anastasia C., Natalia Rodríguez y Carlos E. Rodríguez (2010). «Evaluación in vitro del efecto antibacteriano de los extractos de Bidens pilosa, Lantana camara, Schinus molle y Silybum marianum». En: Revista UDCA Actualidad \& Divulgación Científica 13.2, 117-124. Online: https: / / bit. ly/2GWLFle.

Flores Martínez, L. y col. (2012). «Influencia de las fases lunares sobre el rendimiento del maíz (Zea Mays Variedad NB6)». En: Ciencia e Interculturalidad 10.1, 132-148. Online: https: / / bit.ly / 2GBZRRj.

García Luján, Concepción y col. (2010). «Componentes químicos y su relación con las actividades biológicas de algunos extractos vegetales». En: Química Viva 9.2, 86-96. Online: https:/ / bit. ly/2GBxnXH.

Giraldo, Luz A., Héctor Fabio Ríos y Manuel F. Polanco (2015). «Efecto de dos enraizadores en tres especies forestales promisorias para la recuperación de suelos». En: Revista de Investigación Agraria y Ambiental (RIAA) 1.1, 41-47. Online: https: //bit.ly/2Nik6nz.

Gomes, J\&essica de Andrade y col. (2017). «Physicochemical characteristics and cytotoxic effect of the methanolic extract of Croton heliotropiifo- 
lius Kunth (Euphorbiaceae)». En: African Journal of Pharmacy and Pharmacology 11.28, 321-326. Online: https:/ / bit.ly /2BHEaLd.

González Villa, Ángela A. (2004). «Obtención de aceites esenciales y extractos etanólicos de plantas del Amazonas». Tesis doct. Universidad Nacional de Colombia-Sede Manizales, Online: https://bit.ly/2SU05d1.

Gutiérrez Gaitén, Yamilet I. y col. (2011). «Caracterización química por cromatografía de gases/espectrometría de masas de dos extractos obtenidos de Phyllanthus orbicularis HBK». En: Revista Cubana de Farmacia 45.3, 405-413. Online: https://bit.ly/2GFbd76.

Hammer, Katherine A., Christine F. Carson y Thomas V. Riley (1999). "Antimicrobial activity of essential oils and other plant extracts». En: Journal of applied microbiology 86.6, 985-990. Online: https://bit.ly/2T6Z7cv.

Hernández Ocura, L. y col. (2014). «Potencial Farmacológico de Triterpenos Presentes en Fracciones SólidoLíquido de Euphorbia hyssopifolia L.» En: Revista Científica de la Universidad Autónoma de Coahuila 6.11, 22-27. Online: https: / / bit.ly / 2ElZHLb.

López Ramos, R. y col. (2017). "ANÁLISIS FITOQUÍMICO CUALITATIVO Y EVALUACIÓN DE CAPACIDAD NUTRACÉUTICA DEL EXTRACTO ETANÓLICO DE Croton incanus». En: Investigación y Desarrollo en Ciencia y Tecnología de Alimentos 2, 278-282. Online: https: / / bit.ly / $2 \mathrm{tBtY} 2 \mathrm{p}$.

López Ramosa, Ricardo Guadalupe y col. (2016). «ANÁLISÍS FITOQUÍMICO PRELIMINAR Y EVALUACIÓN DEL EFECTO ANTIMICROBIANO DE HOJA DE Solanum elaeagnifolium.» En: Investigación y Desarrollo en Ciencia y Tecnología de Alimentos 1.1, 226-231. Online: https: / / bit.ly/2tyoA0g.

Muhammad Abubakar, El-Mahmood (2009). «Antibacterial activity of crude extracts of Euphorbia hirta against some bacteria associated with enteric infections». En: Journal of Medicinal Plants Research 3.7, 498-505. Online: https: / / bit.ly / 2BNWBhp.

Mwine, Julius T. y Patrick Van Damme (2011). «Why do Euphorbiaceae tick as medicinal plants? A review of Euphorbiaceae family and its medicinal features». En: Journal of Medicinal Plants Research 5.5, 652-662. Online: https: / / bit. ly/2E2OfmE.
Ogbulie, J. N. y col. (2007). «Antibacterial activities and toxicological potentials of crude ethanolic extracts of Euphorbia hirta». En: African Journal of Biotechnology 6.13, 1545. Online: https: / / bit. ly/2tA7kYc.

Oyerma, C. y col. (2010). «Medicinal plants of Erute county, Lira district, Uganda with particular reference to their conservation». En: African journal of ecology 48.2, 285-298. Online: https: / / bit.ly / 2TaJ914.

Parrales, Adriana, Miguel Reyes y William Pine Tobar (2012). «Cromatografía del Gas Natural». ESCUELA SUPERIOR POLITÉCNICA DEL LITORAL, Online: https://bit.ly/2NkpYfS.

Ramírez, Lucia C., Adriana Castillo y Andrea Vargas (2013). «Evaluación del potencial antibacteriano in vitro deCroton lechleri frente a aislamientos bacterianos de pacientes con úlceras cutáneas». En: NOVA Publicación en Ciencias Biomédicas 11.19, 51-63. Online:/https://bit.ly/2DZL2Ed.

Rodríguez Pava, Cristian Nicolás, Andrés Gabriel Zarate Sanabria y Ligia Consuelo Sánchez Leal (2017). "Actividad antimicrobiana de cuatro variedades de plantas frente a patógenos de importancia clínica en Colombia». En: Nova 15.27, 119-129. Online: https://bit.ly/2TeuyBE.

Sabandar, Carla W. y col. (2013). «Medicinal property, phytochemistry and pharmacology of several Jatropha species (Euphorbiaceae): a review». En: Phytochemistry 85, 7-29. Online: https: / / bit. ly/2SPly6N.

Suzuki, Masashi y col. (2006). «Lanosterol synthase in dicotyledonous plants». En: Plant and Cell Physiology 47.5, 565-571. Online: https:/ / bit.ly / 2tz7LlM.

Torres, Alex (2012). «INFLUENCIA DE LA LUNA EN LA AGRICULTURA». UNIVERSIDAD DE CUENCA FACULTAD DE CIENCIAS AGROPECUARIAS, Online: https://bit.ly/2NmofXj.

Valdéz, Adrián, Ernesto Delgado y Jorge Ramírez (2018). «Actividad adulticida y composición química del aceite esencial de hojas de Lantana camara sobre Drosophila melanogaster». En: Maskana 9.1, 21-30. Online: https:/ / bit.ly/2SOdaV0.

Zampini, Iris Catiana, Norma Cudmani y María Inés Isla (2007). "Actividad antimicrobiana de plantas medicinales argentinas sobre bacterias antibiótico resistentes». En: 41.3, 385-393. Online: https://bit.ly/2GD3k1O. 
Zegarra, Rosario (2015). «Las especies de la familia euphorbiaceae en la provincia de Tacna: estudio biosistemático». En: CIENCIA \& DESARROLLO 19, 44-48. Online: https://bit.ly/2GDSBo0. 